\title{
Healthcare-associated transmission of Panton-Valentine leucocidin positive methicillin-resistant Staphylococcus aureus: the value of screening asymptomatic healthcare workers
}

\author{
Panagiotis Papastergiou ${ }^{1,2^{*}}$ (i) and Eleni Tsiouli, ${ }^{1,2}$
}

\begin{abstract}
Background: Three patients hospitalised in the coronary care unit of a general district hospital (England, UK) were tested positive for Panton-Valentine leucocidin methicillin-resistant Staphylococcus aureus colonisation during their routine weekly screening for methicillin-resistant Staphylococcus aureus (MRSA). The isolates were indistinguishable and all three patients have previously had negative screening tests. The outbreak investigation team considered exploring the possibility of PVL-MRSA transmission from members of staff to the patients and potentially between members of staff.

Method: As part of the investigations, healthcare workers on coronary care unit and intensive care unit were screened for MRSA carriage.

Results: Among 134 screened healthcare workers, five staff members (3.7\%) were MRSA colonised. Among these isolates, four were Panton-Valentine leukocidin positive. However, only two healthcare workers had an indistinguishable isolate with the isolate identified among the colonised patients. Decolonisation treatment was offered to all colonised patients and healthcare workers.

Conclusion: In low MRSA prevalence settings, healthcare workers may be a reservoir of MRSA and an important potential source of transmission to patients. Screening and decolonisation of colonised healthcare workers may provide a valuable strategy in managing linked hospital acquisitions and reduce the risk of occupationally acquired complications. MRSA mass screen of healthcare workers should be considered in transmission with a strain that has a potentially increased virulence, such as Panton-Valentine leucocidin methicillin-resistant Staphylococcus aureus.
\end{abstract}

Keywords: Healthcare worker, Methicillin-resistant Staphylococcus aureus, MRSA, nosocomial, Infection control, Occupational health, Panton-Valentine leucocidin, PVL

\footnotetext{
* Correspondence: panpapast@hotmail.com;

panagiotis.papastergiou@qehkl.nhs.uk; panagiotis.papastergiou@nnuh.nhs.uk

${ }^{1}$ Microbiology Department, NRP Innovation Centre, Norwich Research Park,

Norfolk and Norwich University Hospital, Colney, Norwich NR4 7GJ, UK

${ }^{2}$ Infection Prevention Control/ Microbiology Department, The Queen

Elizabeth Hospital King's Lynn, Gayton Road, King's Lynn PE30 4ET, UK
}

(c) The Author(s). 2018 Open Access This article is distributed under the terms of the Creative Commons Attribution 4.0 International License (http://creativecommons.org/licenses/by/4.0/), which permits unrestricted use, distribution, and reproduction in any medium, provided you give appropriate credit to the original author(s) and the source, provide a link to the Creative Commons license, and indicate if changes were made. The Creative Commons Public Domain Dedication waiver (http://creativecommons.org/publicdomain/zero/1.0/) applies to the data made available in this article, unless otherwise stated. 


\section{Background}

Preventing transmission of methicillin-resistant Staphylococcus aureus (MRSA) is a high priority for infection control and prevention teams. An outbreak of MRSA in healthcare settings can be linked to colonised healthcare workers [1-6].

Panton-Valentine leukocidin (PVL) is an exotoxin transmitted by bacteriophages carried by some MRSA and methicillin sensitive Staphylococcus aureus (MSSA) strains. PVL exotoxin has been associated epidemiologically with virulent, transmissible strains of S. aureus, including community-associated (CA) MRSA [7, 8]. MRSA and PVL-S.aureus infections can cause occupational health problems in healthcare workers (HCWs), including long-term morbidity and mortality $[9,10]$.

All inpatients in our hospital undergo an admission MRSA screen and subsequent once a week MRSA screen which includes the nose, axillae, and any skin lesions.

In September 2016, all patients in our three bedded coronary care unit (CCU) were tested positive during their weekly routine MRSA screen (nose, axilla, groin) three days after their admission to the unit when they were tested negative. This fact indicated that the transmission was more likely to have occurred within this period of three days. The isolates had identical phenotypic susceptibility and were resistant only to beta-lactam antibiotics. All identified MRSA isolates were sent to the Public Health England reference laboratory (Colindale London, UK) for typing. The results showed that all three isolates had indistinguishable staphylococcal protein A (spa) types and spa repeat succession patterns and in addition, they were PVL (Luk-PV) positive. The three bedded CCU is adjoining to the thirteen bedded intensive care unit (ICU) with no door or walls between them and the two units are sharing members of staff. As part of the investigations we identified one more patient in ICU colonised with MRSA from a wound swab two weeks prior to the incident. The ICU patient was discharged one week prior the CCU transmission. This MRSAisolate was proven to be indistinguishable from the one identified among the colonised CCU patients (phenotypical susceptibility, spa type and spa repeat succession patterns).

Mass screening of asymptomatic HCWs for MRSA colonisation is routinely not recommended in the UK. Screening of HCWs is indicated if transmission continues or if the epidemiological aspects of an outbreak are unusual, or if they suggest persistent MRSA carriage by staff [11]. In addition, screening of staff should be performed if a case of PVL-SA infection was acquired in hospital [8].

The outbreak investigation team considered exploring the possibility of PVL-MRSA transmission from members of staff to the patients and potentially between members of staff. Therefore, we contacted an investigation including prospective MRSA testing between HCWs and we present the outcomes of this study.

\section{Methods \\ Enrolment}

A letter was sent to all involved healthcare staff including housekeepers, domestics regular, agency and visiting HCWs from other specialities who have been involved with those patients' care during the three days period between admission to the CCU and MRSA detection. Those were identified by the duty roster and the patient's notes. HCWs were asked to be screened for MRSA carriage in a confidential manner. All staff members on CCU and ICU, including agency staff, housekeepers and domestics were screened for MRSA. In addition, visiting clinicians during the three days period (between admission screen and MRSA positivity) were included in the screening. Samples from nose, axilla, throat and skin rash or wounds (if applicable) were acquired from all HCWs and were sent to the laboratory under a code to ensure pseudonymisation.

\section{Laboratory testing}

Screening swabs were inoculated into Contrast ${ }^{\mathrm{Tm}}$ MRSA broths (Oxoid, UK). Multiple swabs (maximum 4) from the same patient or staff were pooled into a single broth. The samples were processed in the microbiology laboratory of Norfolk and Norwich University Hospital. Staphylococcus aureus ATCC33591 strain was used as a control to monitor the test performance. After incubation at $35-37{ }^{\circ} \mathrm{C}$ for $16-24 \mathrm{~h}$, suspected positive broths and control broths (ATCC33591) were subcultured onto chromogenic Brilliance MRSA-2 agar (Oxoid, UK). Plates were incubated at $37{ }^{\circ} \mathrm{C}$ for $18-20 \mathrm{~h}$. Suspected denim blue colonies were picked onto blood agar for purity. Antibiotic sensitivity testing was performed by EUCAST disk diffusion test methodology. Identification of MRSA isolates was confirmed by VITEK MS (bioMérieux) and antibiotic sensitivities were confirmed by VITEK 2 XL (bioMérieux). The MRSA testing method was applied to both patients and HCWs tested.

All MRSA strains identified at the laboratory were sent for typing to the Antimicrobial Resistance and Healthcare Associated Infections Reference Unit (AMRHAI) in PHE Colindale (London, UK).

\section{Follow up}

The MRSA colonised HCWs were referred to the occupational health department. Expert consultation and decolonisation with octenidine dihydrochloride body wash, mupirocin nasal ointment and mouthwash with $0.2 \%$ chlorhexidine gluconate were offered to the identified MRSA colonised HCWs. The decolonisation was considered successful if the staff member had three consecutive, 
one week apart, negative MRSA screens. Decolonisation of close household member's and the follow-up screens for the discharged patient was overseen by primary care and the PHE authorities.

\section{Results}

Among 134 screened HCWs, five (3.7\%) were colonised with MRSA. The spa typing data showed that the isolates from the $5 \mathrm{HCWs}$ displayed three different spa types and spa repeat succession patterns. Two HCWs were colonised with the epidemic MRSA-PVL isolate that displayed the indistinguishable spa type t019 (spa repeat succession pattern 08-16-02-16-02-25-17-24), identified among all four patients (three CCU patient and one recently discharged ICU patient). Further two HCWs were colonised with a MRSA-PLV isolate that displayed the spa type t002 (spa succession pattern 26-23-17-34-17-20-17-12-17-16). The 5th HCW was colonised with a MRSA isolate that was not PLV positive that displayed the spa type 1843 (spa repeat succession pattern 04-82-17-25-17-25-25-16-17). The number of screened HCWs, CCU, ICU patients and positive PVL-MRSA cases are summarised in Table 1.

\section{Discussion}

Two HCWs were colonised with the MRSA PVL-S.aureus strain spa type t019 identified among the colonised patients, supporting the hypothesis of transmission between patients and healthcare staff.

The prevalence of MRSA colonisation among tested $\mathrm{HCW}$ in the CCU/ICU was 3.7\%. The published MRSA colonisation rates among healthcare staff vary significantly with some countries reporting a relatively high MRSA carriage rate and PVL gene-containing cases among their healthcare staff $[12,13]$. The UK studies conducted in endemic hospital settings report non-outbreak carriage rates between 0 and 15\% [14]. Surveys of healthcare staff at medical institutions in Germany estimate the MRSA prevalence between 0.4 to $4.5 \%$ [15]. Other researchers estimated the prevalence of MRSA in HCWs around $4.6 \%$ and their estimated MRSA prevalence among HCWs in non-outbreak settings was no higher than carriage rates estimated for outbreak [16].

Since all in-patients from high-risk units at the hospital undergo a once weekly routine screen for MRSA, in addition to their admission screen, we were able to determine that the MRSA transmission to these three patients occurred within a relatively short period of $72 \mathrm{~h}$. The fact that two HCWs were tested positive with an indistinguishable isolate suggests that they were part of the transmission. Two weeks before the incidence, a patient repatriated to ICU from another healthcare facility was found to be MRSA colonised. The MRSA was isolated only from the wound site and was not detected from any other site (nose, axilla, groin) during the routine screen and follow up screens. This isolate was proven to be indistinguishable from the one identified among the CCU colonised patients (phenotypical susceptibility, spa type and spa repeat succession patterns). Because the patient's wound site was not initially swabbed during the re-admission, it is not clear if the patients wound was already colonised or became colonised during the re-admission. The ICU patient was discharged a few days before the CCU transmission and no further MRSA cases were detected during the MRSA screens among the ICU patients. The HCWs colonised with the epidemic strain had contact with each other and one HCW had professional contact with the colonised ICU patient and the three CCU patients. Nonetheless, we were unable to identify whether the patient in ICU, two weeks before the incidence, or one of the two HCWs was the "case zero" in this transmission.

Screening of HCWs is recommended if a case of PVL-S.aureus infection was acquired in hospital [8] or if transmission continues or if the epidemiological aspects of an outbreak are unusual, or if they suggest persistent MRSA carriage by staff [11]. In total six patients and staff were colonised with the same indistinguishable PVL-S.aureus isolate, however none among patients or staff had an infection. Exposure to an untreated MRSA carrier is associated with an increase in MRSA acquisition risk and decolonisation can reduce transmission [17].

Occupationally acquired colonisation with methicillinresistant Staphylococcus aureus (MRSA) is an issue of increasing concern that should be addressed. Eradication of colonisation/carriage of healthcare workers should be attempted [11]. In addition, MRSA infection can cause occupational health problems in HCWs that may lead to long-term health problems [10] and occasionally may lead to a fatal outcome [9]. The spread of MRSA isolates with additional potent virulent factors such as PVL require further attention. There is a duty to protect the

Table 1 Number of screened HCWs, CCU, ICU patients and positive PVL-MRSA cases

\begin{tabular}{lllll}
\hline & CCU Patients & ICU Patients & HCWs & Site of screen/ colonisation \\
\hline Patient or HCWs screened & 3 & 13 & 134 & Nose, axilla, groin, wound site (if applicable) \\
PVL-positive MRSA spa type t019 cases & 3 & 1 & 2 & Nose, axilla, groin, wound site \\
PVL-positive MRSA spa type t002 cases & 0 & 0 & 2 & Nose, axilla, groin \\
MRSA (PVL negative) spa type t843 cases & 0 & 0 & 1 & Nose, axilla, groin \\
\hline
\end{tabular}


Table 2 Identified advantages and challenges, during our mass staff screen

\begin{tabular}{ll}
\hline Advantages & Challenges \\
\hline - Epidemiology outbreak investigation - determining the most likely source. & - Testing cost. \\
- Protect the health, safety and welfare of patients, HCWs and their families. & - Time and operational cost for related departments. \\
- Raises awareness among HCWs. & - Fear of stigmatisation if confidentiality and anonymity protection fails. \\
- Improves knowledge. & - Psychological pressure and qualms of conscience among HCWs. \\
- Improves infection control compliance among HCWs. & - Easeless, anxiety for family safety. \\
\hline
\end{tabular}

health, safety and welfare of hospital employees. Irrespectively, if HCWs are potentially the source of an outbreak of MRSA or are colonised with a S.aureus strain that has increased virulence, such as PVL positive, then decolonisation should be considered.

The identified advantages and challenges, during our mass-staff screen, are summarised in Table 2. The information was obtained during compliance observations in ICU/CCU, verbal feedback and discussions with occupational health staff, involved staff representatives and first-hand discussion with affected HCWs.

The letter explaining the concerns and the rationale for the screen was sent to all involved healthcare staff. This provided clarity and contributed significantly to the HCWs engagement in the mass screen process. All identified staff members participated.

The identification of two different MRSA-PVL spa types/ spa repeat succession patterns (t019, 08-16-02-16-0225-17-24 and t002, 26-23-17-34-17-20-17-12-17-16) among the staff was an unexpected finding. Two HCWs were linked with the identified t019 spa type PVL-MRSA transmission. The spa type t019 has been associated with PLV-MRSA belonging to MLST clonal complex 30 reported in the South-West Pacific region and seen previously in the UK [18]. Two further HCWs were asymptomatic carriers of a t002 spa type MRSA that was PVL positive. However, we didn't identify any transmission of that spa type (t002) among patients. There is a possibility that their colonisation was recently acquired abroad. It is also possible that the high hand hygiene and environmental cleaning standards in CCU/ICU, likely reduced further spread.

We considered that potential risk factors for this MRSA transmission was possible fatigue (long shifts) and a mild upper respiratory infection with nasal discharge among one of the MRSA colonised HCW. The risks posed by high workloads can be mitigated by good compliance with infection control measures, training and adequate staffing ratios in the ICU [19]. In addition, it's likely that having a concurrent respiratory infection leads one to be more likely to spread MRSA.

It is worth mentioning that until to date, approximate 21 months later, no further cases of PVL-MRSA have been identified at the CCU and ICU.

Our investigation has limitations. Environmental samples were not obtained since the PVL result was available several days after the transmission occurred and the decontamination of the environment and equipment was completed at CCU and ICU. In addition, we acknowledge that spa typing in MRSA outbreak investigations may be limited due to its low discriminatory power in comparison to whole genome sequencing.

\section{Conclusion}

In conclusion, in low MRSA prevalence settings healthcare workers may be a substantial reservoir of MRSA and an important potential source of transmission to patients. Screening HCWs and decolonisation of colonised members may provide a valuable strategy in managing linked hospital acquisitions and reduce the risk for occupationally acquired complications. MRSA screen and decolonisation should be considered if a healthcare worker is identified as a potential source of a transmission of MRSA. In addition, if healthcare workers are found to be colonised with a S.aureus stain that has a potentially increased virulence, such as PVL toxin, then decolonisation should be offered.

\section{Abbreviations}

CCU: Coronary care unit; HCW: Healthcare workers; ICU: Intensive care unit; MRSA: Methicillin-resistant Staphylococcus aureus; MSSA: Methicillin-sensitive Staphylococcus aureus; PHE: Public Health England; PVL: Panton-Valentine leukocidin

\section{Acknowledgements}

We wish to thank the occupational health staff, ward managers, infection control staff and nursing staff who organised, performed and supported the screening.

\section{Availability of data and materials}

All data generated or analysed during this study are included in this published article.

\section{Authors' contributions}

PP collected results and clinical information, and drafted the initial manuscript. ET commented and critically revised the manuscript for important intellectual content. Both authors read and approved the final manuscript.

\section{Ethics approval and consent to participate}

Written informed consent was not obtained from staff members and approval by the Trust ethics committee was not considered necessary since this was an outbreak investigation and not a research study involving human participants. A letter was sent to all involved healthcare staff and asked to be screened for MRSA carriage. The letter gave assurance that the screen would be done with the utmost regard for confidentiality and aims to maintain the safety of patients and staff. The Occupation Health team carried out the screening and decolonisation treatment in accordance with appropriate NHS Trust protocols and consultant microbiologist advice. 


\section{Competing interests}

Both authors are employees of the Norfolk and Norwich University Hospital NHS Foundation Trust (Norfolk, UK) based at the Queen Elizabeth Hospital King's Lynn (Norfolk, UK). Both authors are appointed in an honorary capacity as a consultant Microbiologist at the Queen Elizabeth Hospital King's Lynn NHS Trust.

\section{Publisher's Note}

Springer Nature remains neutral with regard to jurisdictional claims in published maps and institutional affiliations.

Received: 2 March 2018 Accepted: 21 September 2018

Published online: 27 September 2018

\section{References}

1. Haill C, Fletcher S, Archer R, Jones G, Jayarajah M, Frame J, et al. Prolonged outbreak of meticillin-resistant Staphylococcus aureus in a cardiac surgery unit linked to a single colonized healthcare worker. J Hosp Infect. 2013; 83(3):219-25.

2. Lessing MP, Jordens JZ, Bowler IC. When should healthcare workers be screened for methicillin-resistant Staphylococcus aureus? J Hosp Infect. 1996;34(3):205-10.

3. Lin YC, Lauderdale TL, Lin HM, Chen PC, Cheng MF, Hsieh KS, Liu YC. An outbreak of methicillin-resistant Staphylococcus aureus infection in patients of a pediatric intensive care unit and high carriage rate among health care workers. J Microbiol Immunol Infect. 2007;40(4):325-34.

4. Hart J, Christiansen KJ, Lee R, Heath CH, Coombs GW, Robinson JO. Increased EMRSA-15 health-care worker colonization demonstrated in retrospective review of EMRSA hospital outbreaks. Antimicrob Resist Infect Control. 2014;3(1):7.

5. Méan M, Mallaret MR, Andrini P, Recule C, Debillon T, Pavese P, Croizé J. A neonatal specialist with recurrent methicillin-resistant Staphylococcus aureus (MRSA) carriage implicated in the transmission of MRSA to newborns. Infect Control Hosp Epidemiol. 2007;28(5):625-8.

6. Kampf G, Adena S, Rüden H, Weist K. Inducibility and potential role of MecA-gene-positive oxacillin-susceptible Staphylococcus aureus from colonized healthcare workers as a source for nosocomial infections. J Hosp Infect. 2003;54(2):124-9.

7. Watkins RR, David MZ, Salata RA. Current concepts on the virulence mechanisms of meticillin-resistant Staphylococcus aureus. J Med Microbiol. 2012;61:1179-93.

8. Health Protection Agency. Guidance on the diagnosis and management of PVL-associated Staphylococcus aureus infections (PVL-SA) in England, 2nd ed. London: Health Protection Agency; 2008. https://assets. publishing.service.gov.uk/government/uploads/system/uploads/ attachment_data/file/322857/Guidance_on_the_diagnosis_and_ management_of_PVL_associated_SA_infections_in_England_2_Ed.pdf. Accessed 19 Dec 2017

9. Orendi JM, Coetzee N, Ellington MJ, Boakes E, Cookson BD, Hardy KJ, et al. Community and nosocomial transmission of Panton-Valentine leucocidinpositive community-associated meticillin-resistant Staphylococcus aureus: implications for healthcare. J Hosp Infect. 2010;75(4):258-64.

10. Haamann F, Dulon M, Nienhaus A. MRSA as an occupational disease: a case series. Int Arch Occup Environ Health. 2011;84(3):259-66.

11. Coia JE, Duckworth GJ, Edwards DI, Farrington M, Fry C, Humphreys H, Mallaghan C, Tucker DR. Guidelines for the control and prevention of meticillin-resistant Staphylococcus aureus (MRSA) in healthcare facilities. J Hosp Infect. 2006;63(Suppl 1):S1-44.

12. Rastegar Lari A, Pourmand MR, Ohadian Moghadam S, Abdossamadi Z, Ebrahimzadeh Namvar A, Asghari B. Prevalence of PVL-containing MRSA isolates among hospital staff nasal carriers. LabMedicine. 42:283-6.

13. Shibabaw A, Abebe $T$, Mihret A. Nasal carriage rate of methicillin resistant Staphylococcus aureus among Dessie referral hospital health care workers; Dessie, Northeast Ethiopia. Antimicrob Resist Infect Control. 2013;2(1):25.

14. Hawkins G, Stewart S, Blatchford O, Reilly J. Should healthcare workers be screened routinely for meticillin-resistant Staphylococcus aureus? A review of the evidence. J Hosp Infect. 2011;77(4):285-9.

15. Ruscher C. Empfehlungen zur Prävention und Kontrolle von Methicillinresistenten Staphylococcus aureus-Stämmen (MRSA) in medizinischen und pflegerischen Einrichtungen-Empfehlung der
Kommission für Krankenhaushygiene und Infektionsprävention (KRINKO) beim Robert Koch-Institut. Bundesgesundheitsblatt Gesundheitsforschung Gesundheitsschutz. 2014;57:695-732.

16. Dulon M, Peters C, Schablon A, Nienhaus A. MRSA carriage among healthcare workers in non-outbreak settings in Europe and the United States: a systematic review. BMC Infect Dis. 2014;14:363.

17. Pierce R, Lessler J, Popoola VO, Milstone AM. Meticillin-resistant Staphylococcus aureus (MRSA) acquisition risk in an endemic neonatal intensive care unit with an active surveillance culture and decolonization programme. J Hosp Infect. 2017:95:91-7.

18. Ali H, Nash JQ, Kearns AM, Pichon B, Vasu V, Nixon Z, Burgess A, Weston D, Sedgwick J, Ashford G, Mühlschlegel FA. Outbreak of a south West Pacific clone Panton-Valentine leucocidin-positive meticillin-resistant Staphylococcus aureus infection in a UK neonatal intensive care unit. J Hosp Infect. 2012;80:293-8.

19. Kong F, Cook D, Paterson DL, Whitby M, Clements AC. Do staffing and workload levels influence the risk of new acquisitions of meticillin-resistant Staphylococcus aureus in a well-resourced intensive care unit? J Hosp Infect. 2012;80:331-9.

\section{Ready to submit your research? Choose BMC and benefit from:}

- fast, convenient online submission

- thorough peer review by experienced researchers in your field

- rapid publication on acceptance

- support for research data, including large and complex data types

- gold Open Access which fosters wider collaboration and increased citations

- maximum visibility for your research: over $100 \mathrm{M}$ website views per year

At BMC, research is always in progress.

Learn more biomedcentral.com/submissions 